Research Paper

\title{
Phase I/II Trial Evaluating Carbon lon Radiotherapy for Salvaging Treatment of Locally Recurrent Nasopharyngeal Carcinoma
}

\author{
Lin Kong1, Jiyi $\mathrm{Hu}^{2}$, Xiyin Guan², Jing $\mathrm{Gao}^{2}$, Rong $\mathrm{Lu}^{3}$, Jiade J. $\mathrm{Lu}^{2}{ }^{\circledR}$ \\ 1. Department of Radiation Oncology, Shanghai Proton and Heavy Ion Center, Fudan University Shanghai Cancer Center, Shanghai, China; \\ 2. Department of Radiation Oncology, Shanghai Proton and Heavy Ion Center, Shanghai, China; \\ 3. Department of Outpatient Clinic, Shanghai Proton and Heavy Ion Center, Shanghai, China.
}

$\triangle$ Corresponding author: Jiade J. Lu, M.D., M.B.A. Address: Shanghai Proton and Heavy Ion Center, 4365 Kangxin Road, Pudong, Shanghai, 201315, China. Email: jiade.lu@sphic.org.cn Tel: +86-21-38296518.

(C) Ivyspring International Publisher. Reproduction is permitted for personal, noncommercial use, provided that the article is in whole, unmodified, and properly cited. See http://ivyspring.com/terms for terms and conditions.

Received: 2015.11.12; Accepted: 2016.02.11; Published: 2016.04.10

\begin{abstract}
Background: Radiation therapy is the mainstay strategy for the treatment of nasopharyngeal cancer (NPC). Intensity-modulated X-ray therapy (IMXT) alone is the current standard for stage I and II NPC. For stage III and IV A/B diseases, concurrent chemotherapy should be provided in addition to IMXT. However, optimal treatment for locally recurrent NPC after previous definitive dose of radiotherapy is lacking. Various techniques including brachytherapy, IMXT, stereotactic radiosurgery or radiotherapy (SRS or SBRT) have been used in the management of locally recurrent NPC. Due to the inherent limitation of these techniques, i.e., limited range of irradiation or over-irradiation to surrounding normal tissues, moderate efficacy has been observed at the cost of severe toxicities. Carbon ion radiotherapy (CIRT) offers potential physical and biological advantages over photon and proton radiotherapy. Due to the inverted dose profile of particle beams and their greater energy deposition within the Bragg peak, precise dose delivery to the target volume(s) without exposing the surrounding organs at risk to extra doses is possible. In addition, CIRT provides an increased relative biological effectiveness (RBE) as compared to photon and proton radiotherapy. Such advantages may translate to improved outcomes after irradiation in terms of disease control in radio-resistant and previously treated, recurrent malignancies. It is therefore reasonable to postulate that recurrent NPC after high-dose radiotherapy could be more resistant to re-irradiation using photons. Reports on the treatment of radio-resistant malignancies in the head and neck region such as melanoma, sarcoma, and adenoid cystic carcinoma (ACC) have demonstrated superior local control rates from CIRT as compared to photon irradiation. Thus patients with recurrent NPC are likely to benefit from the enhanced biological effectiveness of carbon ions. As effective retreatment strategy is lacking for locally recurrent NPC, carbon ion radiation therapy offers an ideal alternate to conventional X-ray irradiation.

Methods and Design: The recommended dose of re-irradiation using CIRT for locally recurrent NPC will be determined in the dose-escalating phase (Phase I) of the study. Efficacy in terms of local progression-free survival (LPFS) and overall survival (OS) will be studied in the second phase of the study. Increasing doses of CIRT using raster scanning technology from 55GyE $(22 \times 2.5 \mathrm{GyE})$ to $65 \mathrm{GyE}(26 \times 2.5 \mathrm{GyE})$ will be delivered in the Phase I part of the study. The primary endpoint of the Phase I part of the study is acute and sub-acute toxicities; the primary endpoint in the Phase II part is local progression-free survival and overall survival. Using the historical 2-year OS rate of $50 \%$ in locally recurrent NPC patients treated with photon or proton, we hypothesize that CIRT can improve the 2 -year OS rate to $70 \%$.
\end{abstract}


Discussion: The utilization of conventional radiation techniques including IMXT, brachytherapy, or stereotactic radiation therapy provides moderate efficacy in the treatment of locally recurrent NPC due to the limitations in dose distribution and biological effectiveness. Improved outcome in terms of treatment-induced toxicity, LC, LPFS, and OS are expected using CIRT due to the physical and biological characteristics of carbon ion beam. However, the recommended dose of CIRT used in re-irradiation for the local NPC focus remain to be determined. The recommended dose as well as the efficacy of CIRT in the treatment of locally recurrent NPC will be evaluated in the present trial.

Key words: Carbon Ion Radiotherapy, recurrent nasopharyngeal cancer

\section{Introduction}

Nasopharyngeal cancer (NPC) is the most commonly diagnosed head and neck malignancy and endemic in Southern China, and radiation therapy is the only curative treatment modality for non-metastatic NPC. The prevailing use of intensity-modulated photon based radiation therapy (IMXT) has significantly improved the treatment outcomes including local and regional control ${ }^{1}$. However, approximately $10 \sim 15 \%$ patients will suffer from local recurrence despite of aggressive treatment.

Although surgery (i.e., nasopharyngectomy) has been used in a selected group of patients with limited disease volume, re-irradiation remains the principle modality for patients with locally recurrent NPC 2,3,4. Various strategies, including brachytherapy, stereotactic radiosurgery (SRS), and IMXT have been utilized in an attempt to control local recurrent NPC. However, although brachytherapy and SRS can usually sufficiently spare the organs at risk (OARs) surrounding the disease foci, their utilization is usually limited by the extent of disease. The use of IMXT has been reported mainly from the endemic area. Several groups in China have reported the long-term results in patients re-irradiated with IMXT for their patients diagnosed with locally recurrent NPC5,6,7. The documented long-term overall survival (OS) rates range from $45 \sim 65 \%$, and patients with more advanced disease at recurrence suffer from far a more dismal outcome, usually with an OS rate of $<40 \%$.

\section{Challenges in the Re-treatment of NPC Local Recurrence}

Treatment of locally recurrent NPC after high-dose radiation is challenging for several reasons. The high-dose areas of the initial radiation therapy for primary disease usually encompass not only the gross tumor volume but also the surrounding OARs such as nasopharyngeal mucosa, temporal lobes of the brain, brain stem, and optic nerve/chiasm for subclinical disease. A substantial portion of the temporal lobes of the brain and most parts if not the entire nasopharyx may be covered by a high dose of $>66$ Gy especially in T3 or T4 NPC. The incidence of severe adverse effects
(SAEs) increases significantly when the combined radiation dose from initial and re-irradiation exceeds $100 \mathrm{~Gy}^{8}$. Thus, re-irradiation to a dose of 60Gy or more may cause long-term radiation-induced SAEs such as brain necrosis and mucosal ulceration and necrosis. Mucosal ulceration and necrosis is a devastating $\mathrm{SAE}$, which will directly cause a significant loss of quality of life, even death due to infection or massive hemorrhage of the internal carotid artery.

Local recurrence after high-dose photon radiotherapy may be secondary to photon resistant cancer cells that survived initial course of treatment. NPC patients might also fail locally due to a marginal miss after $2 \mathrm{D}$ or $3 \mathrm{D}$-conformal radiation therapy (3D-CRT). The prevailing utilization of IMXT has significantly improved the treatment outcome especially local and regional disease control. The utilization of advanced diagnostic technology such as MRI and PET/CT together with IMXT have been expected to minimize the instances of marginal misses of the primary disease and neck adenopathy. However, approximately $10 \sim 15 \%$ of patients completed IMXT of 70Gy still fail locally. Therefore, it is reasonable to postulate that subgroups of cells within the gross disease may be more resistant to photon radiotherapy. Whether such features of radio-resistance is caused by hypo-oxygenation, inherently resistant clones, or the presence of cancer stem cells (CSCs) or stem cell-like cells remain to be investigated. Nevertheless, their characteristics of radio-resistance to X-ray based IMXT could confer a more dismal outcome after re-irradiation using IMXT. Salvage radiation treatment using photon therapy to the same or lower dose, usually in the range between 60 70Gy at standard fractionation, may not produce sufficient long-term disease control. As such, previous treatment using IMXT in NPC poses additional challenges to re-irradiation for local recurrence as this clinical scenario requires the targeting of potential radio-resistant disease while in the setting of OARs which have already received significant doses. 


\section{Characteristics of Proton and Carbon Ion in Cancer Treatment}

Particle therapy such as proton or carbon-ion radiation therapy (PRT or CIRT) provides distinct physical characteristics include: a sharp lateral penumbra; very low energy deposition within the entry path prior to the Bragg peak formed by the steep dose deposition; and, a sharp dose fall-off after the Bragg peak, thus possessing a dose delivery with a finite range. The depth of the Bragg peak is determined by the beam energy. Spearing of normal surrounding tissues is crucial in radiation therapy of head and neck area especially patients who have completed a previous course of high-dose radiation. A number of studies have reported superior dose distributions using particle therapy for primary or recurrent NPC with acceptable clinical outcomes, ${ }^{9,10,11}$.

In addition to its superior physical properties, carbon ion is a high LET modality and the relative biological effectiveness (RBE) of CIRT is significantly higher than those of photon and proton radiation. The value of $R B E$ is $3 \sim 5$ for carbon ion depend on the tissue type and end point of study. It has been suggested that more damage from high LET radiation is in the form of direct DNA double strand breaks, which is more difficult to repair ${ }^{12}$. As such, improved clinical results could be expected after high-LET radiation such as CIRT especially for photon-resistant cancer cells. As per convention with CIRT (and other particle-based modalities), we will account for the RBE and LET differences between CIRT and photon radiotherapy and report our CIRT doses in terms of Gray Equivalents (GyE), which refer the biologic equivalent doses of photons.

\section{PT for Local Recurrence in the Base of Skull}

Improved treatment results from CIRT have been demonstrated for a number of disease entities including chordoma/chondrosarcoma of the skull base, melanoma and adenoid cystic carcinomas (ACC) of the head and neck area13,14,15,16. CIRT is routinely offered to patients of tumor entities at the Heidelberg Ion-Beam Therapy Center (HIT), Germany and the National Institute of Radiological Science (NIRS), Japan. Safety of CIRT to critical OARs such as optical nerve/chiasm, brain, brainstem, or spinal cord, has also been reported.

Particle therapy has been used in the re-treatment of local recurrence of head and neck tumors including NPC. In a retrospective study of 16 locally recurrent NPC patients re-irradiated with proton therapy, the authors found that dose of 61Gy or more (in 1.8-2.0 GyE per daily fraction) produced a local control of $60 \%$ vs. $38 \%$ for those who received less total dose $(\mathrm{P}=0.17)^{11}$. The overall survival (OS) at
24 months was $50 \%$. With a cumulative dose of 133.5Gy (range, 110-148) from for both initial and repeated radiation, 2 of $9(22.2 \%)$ surviving patients developed grade 3 or 4 late toxicity (osteo- and mucosal necrosis) which were successfully treated. The doses to the optic chiasm, surface and center of the brainstem were $60.0 \mathrm{~Gy}, 60.0 \mathrm{~Gy}$, and 57.0 Gy, respectively, but no patient experienced neurological deficit. The BED2 and BED9 of the re-irradiation regimen were 116.3GyE and 61.2GyE, respectively for late responding tissues and tumor tissue.

A more recently published series from HIT studied a group of 28 patients with local recurrence after initial radiation therapy. Eighteen patients had skull of base tumors and were mostly treated with carbon re-irradiation to $51 \mathrm{GyE}$ in $3 \mathrm{GyE}$ daily fractions over 17 days, 7 days a week (range, 42 60GyE) ${ }^{17}$. Three of the 18 patients received 2 courses of radiotherapy prior to the re-irradiation using carbon for their recurrence. Grade 1 or 2 early or late toxicity were only observed in 5 patients. No patient developed Grade 3 or 4 early or late toxicities. An alpha/beta value of 2 was used for the endpoint late toxicity to the normal tissue. Therefore, the BED2 and BED9 were 127.5GyE and 68GyE, respectively.

With the superior physical and biological properties of CIRT, it is reasonable to postulate that CIRT may produce promising outcome in locally recurrent NPC after IMXT. In this current phase I/II study, we first intend to define the maximal tolerated dose (MTD) of CIRT for re-irradiation for NPC patients with local failure after their initial course of photon-based intensity-modulated radiotherapy, then test the efficacy of the defined MTD.

\section{The Selection of CIRT Dose for Re-irradiation in the Phase I Study}

In the phase I stage of the study, the recommended dose (RD) for re-irradiation using CIRT for locally recurrent NPC will be determined in a dose escalation scheme. The maximal tolerated dose (MTD) will be determined using a TITE-CRM design 18,19 . The efficacy of the RD will then be investigated in the phase II part of this trial, of which the results will be compared to historical controls treated with IMXT with or without combined chemotherapy. Patients will be monitored for local control, progression-free survival, and overall survival as well as adverse-effects.

In the above-mentioned studies that used IMXT for the re-treatment of NPC recurrence, doses ranging from 60-70Gy in conventional fractionation have been used. However, such dose is associated with $\sim 35 \%$ Grade 3 or 4 late toxicity including nasopharyngeal ulceration $(\sim 20 \%)$ and hemorrhage, especially at 
higher dose approximates 70Gy [5]. In a series of 239 patients treated to a mean dose of 70Gy (range $61.73 \sim 77.54 \mathrm{~Gy})$ to GTV, the cause of death of the $69.2 \%$ of the 120 deceased patients (34.7\% of the entire group of patients), was radiation injuries [6]. Currently, the most commonly selected dose of IMXT for re-treatment of NPC local recurrence ranges between 60 66Gy (i.e., BED 73 81GyE for alpha/beta of 9). However, long-term results after such treatment especially in patients recurred after previous IMXT for initial treatment is lacking.

Accepted long-term toxicity were reported in the 2 published papers on re-irradiation using PT. A BED2 of approximately $120 \mathrm{GyE}$ was delivered to the recurrent tumor associated with $22 \%$ and 0 Grade $3 / 4$ late toxicity, in these respective reports. Using a daily fraction of $2.5 \mathrm{GyE}$, a total dose of $55 \mathrm{GyE}$ in 22 daily fractions will provide BED2 of 124GyE, and BED9 of 70GyE. As we are seeking an MTD that produces < $25 \%$ probability of severe acute or sub-acute toxicity (defined as any Grade 4 acute or subacute toxicity possibly, probably, and definitely induced by CIRT observed within 6 month after the completion of treatment, except for Grade 4 neutropenia without fever), such dose and fractionation, i.e., 57.5 GyE at 2.5GyE/day serves a good starting dose/fractionation based on historical data.

Because of the sharper penumbra offered by CIRT, we anticipate that we will be able to deliver higher doses to our clinical targets while potentially lowering the integral dose to and high-dose volumes within our non-targeted structures. Therefore, in addition to determining a maximally tolerated dose, we also hope to correlate toxicity to various other dosimetric characteristics, which account for the more rapid dose falloff of CIRT such as: dose-volume histogram, gradient indices and conformality indices of the various biologic effective doses. This will provide additional information to the previously-treated tissue tolerance of CIRT.

\section{Methods and Design}

This is a single-center Phase I/II single arm clinical trial with the purpose of determining the MTD of re-irradiation with CIRT in the treatment of locally recurrent nasopharyngeal cancer (NPC) and evaluating the efficacy of such treatment at the determined MTD.

\subsection{Objectives}

\section{Phase I}

The primary objective is defining the MTD for the subsequent phase of the trial by observation of the severe acute or sub-acute toxicities, defined as Grade 4 or above according to CTCAE v. 4.03 possibly, probably, or definitively induced by re-irradiation using CIRT within 6 months from the completion of CIRT. The secondary objective is to evaluate the LPFS and OS after re-irradiation using CIRT.

\section{Phase II}

The primary endpoint is the 2-year overall survival (OS) after re-irradiation with CIRT. The secondary endpoints are imaging response, local control (LC), disease-free survival (DFS), and long-term toxicity and safety.

\subsection{Trial design and schedule}

This is a single-center single-arm Phase I/II clinical trial.

\section{Phase I: Dose Escalation to Determine MDT}

The aim of the first phase of the trial is to determine the MTD of re-irradiation with CIRT using raster-scanning technology for locally recurrent NPC. Patients meet the inclusion criteria of the trial will be treated with this CIRT of escalating dose regimens to evaluate the maximal tolerated dose (MTD) in terms of acute and subactue toxicity observed during and within 6 months after the completion of CIRT.

Patients will be treated within 5 increasing dose regimens starting at $55 \mathrm{GyE}(2.5 \mathrm{GyE} \times 20$ fractions $)$ up to 65 GyE (2.5 GyE $\times 26$ fractions) using the TITE-CRM design of Phase I trial with the starting dose/fractionation at 57.5GyE delivered 23 daily fractions. Dose levels, dose fractionation, and estimated probability for DLT (pDLT) are detailed in Table 1. The rationales for this starting dose were detailed in the Introduction of this protocol. If the MTD is not reached at $65 \mathrm{GyE}$, further dose escalation will not be considered based on historical data on dose-toxicity association, and $65 \mathrm{GyE}$ will be considered as the recommended dose that will be tested in the Phase II trial.

Table 1. Treatment schedule for dose escalation.

\begin{tabular}{|c|c|c|c|c|c|}
\hline $\begin{array}{r}\text { Dose } \\
\text { Level } \\
\end{array}$ & Dose and fractionation & Total dose & pDLT & $\begin{array}{l}\text { BED2 } \\
\text { (GyE) }\end{array}$ & $\begin{array}{l}\text { BED9 } \\
\text { (GyE) }\end{array}$ \\
\hline 1 & 22 x $2.5 \mathrm{GyE}$ & $55 \mathrm{GyE}$ & $<5 \%$ & 123.8 & 70.3 \\
\hline 2 & 23 x 2.5 GyE (Starting Dose) & $57.5 \mathrm{GyE}$ & $10 \%$ & 129.4 & 73.5 \\
\hline 3 & $24 \times 2.5 \mathrm{GyE}$ & $60 \mathrm{GyE}$ & $20 \%$ & 135.0 & 76.7 \\
\hline 4 & $25 \times 2.5 \mathrm{GyE}$ & $62.5 \mathrm{GyE}$ & $30 \%$ & 140.6 & 79.9 \\
\hline 5 & $26 \times 2.5 \mathrm{GyE}$ & $65 \mathrm{GyE}$ & $40 \%$ & 146.3 & 83.1 \\
\hline
\end{tabular}

A maximum of 25 patients for 5 dose levels are projected for the dose-escalating part of the trial. We expect to recruit 2 patients per months on average. Since the first patients of each dose level will be followed up for 6 months before dose escalating to the next level, and all patients will be followed up for at 
least 6 months before the closure of the phase I part of the study, the phase I part is expected to be completed in 24 months.

For ethical considerations, all patients treated in the Phase I part of this trial at the recommended dose (i.e., MTD) will be included in the Phase II part of the study to minimize the number of patients to be recruited.

\section{Phase II: Treatment at Recommended Dose to Study Efficacy}

Once the MTD for locally recurrent NPC is determined, the MTD will be used as the recommended dose to patients fulfilling the inclusion criteria in the Phase II part of the trial. If MTD cannot be determined, 65GyE will be used to treat the patients in the Phase II part of the study.

The endpoints are overall survival (OS) after re-irradiation at 24 months (median follow-up). Therefore, all patients will be followed up for at least 12 months or until death to evaluate the primary outcomes of Phase II. The estimated overall duration of the Phase 2 part of the trial is approximately 24 months. Recruitment of the patients is planned over 12 months for a total of 40 patients (less the number of patients treated at MTD in Phase 1). Minimum duration of the follow-up phase will be 12 months for all patients in the phase 2 part of the study.

\subsection{Inclusion and Exclusion Criteria}

Patients with the diagnosis of locally recurrent NPC will be evaluated and screened for the study. All patients fulfilling the inclusion/exclusion criteria will be notified with the details of this clinical trial. Inclusion and exclusion criteria are detailed in Table 2.

Table 2. Inclusion and exclusion criteria.

\begin{tabular}{|c|c|}
\hline Inclusion Criteria & Exclusion Criteria \\
\hline $\begin{array}{l}\text { * Willing to sign the written } \\
\text { informed consent; Informed consent } \\
\text { must be signed before the enrolment } \\
\text { in the trial } \\
\text { * Pathologically and clinically } \\
\text { confirmed locally recurrent NPC } \\
\text { * Completed a definitive course of } \\
\text { IMXT to a total dose of } \geq 66 \text { Gy } \\
\text { * Recurrence diagnosed more than } \\
12 \text { months after the initial course of } \\
\text { IMXT } \\
\text { * Age } \geq 18 \text { and }<70 \text { years of age } \\
\text { * Karnofsky Performance Score } \geq 70 \\
\text { * Willing to accept adequate } \\
\text { contraception for women with } \\
\text { childbearing potential } \\
\text { * Ability to understand character } \\
\text { and individual consequences of the } \\
\text { clinical trial }\end{array}$ & $\begin{array}{l}{ }^{*} \text { Refusal of the patient to participate } \\
\text { into the study } \\
\text { Presence of distant metastasis } \\
\text { Local recurrence of NPC diagnosed } \\
\text { within } 12 \text { months from the completion } \\
\text { of previous course of radiation therapy } \\
\text { * Technology used other than IMXT } \\
\text { (including brachytherapy following } \\
\text { IMXT) for the treatment of initial } \\
\text { diagnosis of NPC } \\
\text { * Pregnant or lactating women } \\
\text { Patients who have not yet recovered } \\
\text { from acute toxicities of prior therapies } \\
\text { * A diagnosis of malignancy other than } \\
\text { CIS of the cervix, BCC and SCC of the } \\
\text { skin within the past } 5 \text { years } \\
\text { * Being included in other clinical } \\
\text { studies of which treatments may } \\
\text { interfere the outcome of the current } \\
\text { trial }\end{array}$ \\
\hline
\end{tabular}

\subsection{Induction, concurrent, and adjuvant treatments}

\subsubsection{Induction chemotherapy}

Neoadjuvant chemotherapy will be used based on the stage of the recurrent NPC. Chemotherapy and/or targeted therapy will be provided to patients with rT3, rT4, or rN+ diseases except for those with retroperitoneal lymph node recurrence and completed prior to the initiation of CIRT. Patients with rT1, rT2, or recurrent retropharyngeal node disease only are not required to receive mandatory chemotherapy.

\subsubsection{Concurrent chemotherapy}

Concurrent chemotherapy or any other anti-tumor treatments are not allowed with CIRT. Adjuvant chemotherapy is not allowed unless local recurrent, progression or distant metastasis after the completion of CIRT is evident.

\subsubsection{Other medications}

Only medication needed for inter-concurrent diseases (i.e., diabetes, hypertension, hyperlipidemia, benign prostate hyperplasia, etc) of the patients are allowed during the re-irradiation of patients. The use of such medication(s) must be discussed with the principal investigator prior to the inclusion to the trial.

\subsection{Radiation Therapy}

\subsubsection{Treatment planning}

Patients will be registered and immobilized using an individual immobilization system for both planning and treatment. Treatment planning will be performed about 10 working days prior to the start of CIRT. Planning CT without contrast will be performed and MRI taken in treatment position will be obtained and fused with planning CT. As all patients included in this study will have completed photon RT of 66 Gy or above, organs at risk such as the brain stem, optic nerve and chiasm, temporal lobes of the brain, and eyes will be contoured. Discount of the doses to the OARs from the initial radiation course was uniformly set at $70 \%$, i.e., $30 \%$ residual doses were used to calculate the limiting dose to the OARs. Dose limitations of OARs will be controlled according to Emami et al ${ }^{20}$.

- Gross Tumor Volume (GTV) - will be defined as the gross disease seen on the planning $\mathrm{CT}$, area of contrast enhancement on T1-weighted MRI, and lesion(s) with high SUV uptake observed on FDG-PET/CT (optional)

- Clinical Target Volume (CTV) - CTV for gross tumor will be defined as the GTV $+3 \sim 5 \mathrm{~mm}$ 
margin; the CTV for subclinical disease will be defined based on the clinical judgment for potential subclinical disease.

- Planning Target Volume (PTV) - will be added depending on individual factors such as patient positioning or beam angles chosen and will range $3 \sim 6 \mathrm{~mm}$

CIRT planning is performed using the Syngo treatment planning system (Siemens, Erlangen, Germany) including biologic plan optimization. Biologically effective dose distributions will be calculated using the $a / \beta$ ratio of 9 for nasopharyngeal cancer and 3 for late toxicity, respectively.

\subsection{2a Dose prescription for CIRT}

Treatments will be delivered using the IONTRIS intensity-modulated raster scan system.

Five dose levels and their initial estimation of the probability for DLT (pDLT) are planned within the Phase I part (Table 1). Daily fraction of $2.5 \mathrm{GyE}$ will be delivered up to the total dose for all dose levels in the dose escalation phase I. After the recommended dose $(\mathrm{RD})$ is determined or if the treatments to $65 \mathrm{GyE}$ are safely delivered, the recommended dose (or $65 \mathrm{GyE}$ ) will be the prescribed dose in the Phase II part of the study.

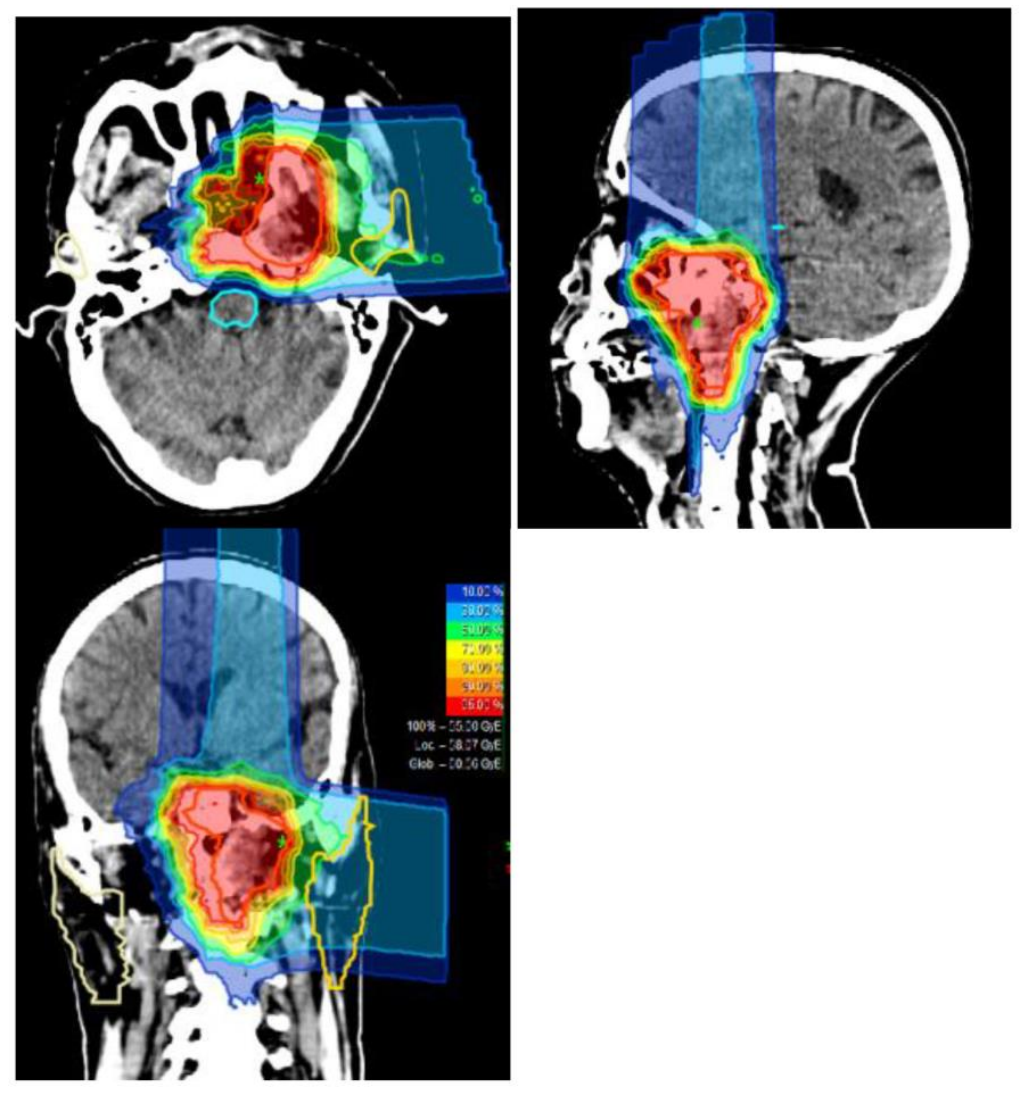

Figure 1. Typical treatment plan and dose distribution for carbon ion radiotherapy delivered using the raster-scanning technique in a patients with locally recurrent nasopharyngeal carcinoma.
Ninty-five percent (95\%) of the isodose line should cover the CTV for gross tumor (i.e., GTV+3 5mm) and 90\% dose line should cover the PTV for gross disease. The CTV for other subclinical disease, if applicable, should be irradiated to $90 \%$ of the assigned dose level.

Dose specification is based on biologic equivalent dose (BED) because the relative biological effectiveness (RBE) of CIRT differs significantly from photon therapy and within the target volume. The dose prescription used is related to the isoeffective dose GyE (Gray equivalent) for 5 daily fractions per week at 2 Gy per fraction.

\subsection{2b Dosimetric recording of non-targeted structures CIRT}

Biologic equivalent dose delivered to the non-targeted structures and organs at risk will be quantified with respect to maximum point dose, absolute dose-volumes, percent dose-volumes, structural dose gradient and conformality index of the treatment plan will be recorded and used for later correlation with the development of any toxicities.

\subsubsection{Treatment planning and delivery}

CIRT in the form of intensity modulated carbon-ion radiation therapy (IMCT) will be delivered using raster-scanning technology. Patients are treated for once a day, 5 days per week. Treatment interruptions of more than 3 days are not allowed unless severe adverse effects require so. Treatment during weekends are required if a break of 2 or more days occurred during the working days. Patient positioning prior to CIRT will be evaluated by comparison of orthogonal $\mathrm{x}$-rays to the DRRs. Set up deviations $>2$ $\mathrm{mm}$ are corrected prior to treatment delivery. A typical treatment plan is illustrated in Figure 1.

\subsection{Assessment of Efficacy Parameters}

\subsubsection{Baseline documentation of "target" lesion}

The target lesion is defined as the gross tumor locally recurred in the post-nasal space delineated for re-irradiation using CIRT. A sum of the longest diameter (LD) for the gross tumor will be measured and reported as the baseline sum LD. The baseline sum LD will be used as reference by which to characterize the objective tumor. 


\subsubsection{Response to Treatment}

Response to CIRT will be recorded according to the RECIST criteria.

Evaluation of the target lesion per RECIST criteria

- Complete Response (CR): Complete disappearance of the target lesions

- Partial Response (PR): At least a 30\% decrease in the sum of the LD of the target lesion, taking as reference the baseline sum LD

- Stable Disease (SD): Neither sufficient shrinkage to qualify for PR nor sufficient increase to qualify for PD, taking as reference the smallest sum LD since the treatment started

- Progressive Disease (PD): At least a 20\% increase in the sum of the LD of the target lesion, taking as reference the smallest sum LD recorded since the treatment (including induction chemotherapy) started

Evaluation of non-target lesion(s)

- Complete Response (CR): Disappearance of all non-target lesions and normalization of tumor marker level (N/A for the current study)

- Incomplete Response/Stable Disease (SD): Persistence of one or more non-target lesion(s) or/and maintenance of tumor marker level above the normal limits (N/A for the current study)

- Progressive Disease (PD): Appearance of one or more new lesions and/or unequivocal progression of existing non-target lesions

Evaluation of best overall response

The best overall response is the best response recorded from the start of the treatment until disease progression/recurrence (taking as reference for PD the smallest measurements recorded since the treatment started). In general, the patient's best response assignment will depend on the achievement of both measurement and confirmation criteria

- Patients with a global deterioration of health status requiring discontinuation of treatment without objective evidence of disease progression at that time should be classified as having "symptomatic deterioration". Every effort should be made to document the objective progression even after discontinuation of treatment.

- In some circumstances it may be difficult to distinguish residual disease from normal tissue. When the evaluation of complete response depends on this determination, it is recommended that the residual lesion be investigated (fine needle aspirate/biopsy) to confirm the complete response status.

\subsubsection{Overall Survival}

The duration of overall survival is the time interval between the pathological diagnosis of NPC local recurrence and the date of death due to any cause. Patients not reported deceased or lose to follow-up will be censored at the date of the last follow-up session.

\subsubsection{Progression-free survival}

Progression-free survival (PFS) is the time interval between the completion of CIRT and the dated of disease recurrence or progression at any body part. Patients not reported dead or lost to follow- up will be censored at the date of the last follow-up examination.

\subsection{Assessment of Toxicity/Safety and Other Parameters}

\subsubsection{Toxicity and Safety Parameters}

Common Terminology Criteria for Adverse Events (CTCAE) version 4.03 will be used for toxicity and adverse event evaluation and reporting. Safety and toxicity of the CIRT will be evaluated at each weekly on-treatment examination during radiotherapy and at each follow-up by physical examination and lab tests.

\subsubsection{Other Parameters}

Peripheral blood will be collected for measuring the EBV DNA and/or other tumor markers. Such parameters may be used to predict the extent of disease or treatment outcome, or for other research purposes may or may not be directly related to the current trial. A separate informed consent is required from the patients.

\subsection{Follow-up after the completion of the trial}

\subsubsection{Follow-up}

After completion of the study treatment, patients will be followed up regularly according to the following schedule and plan indefinitely or until death. The first and second follow-up will be scheduled at 1 and 3 months after the completion of CIRT. Patients are then scheduled for follow-up visit every 3 months or as clinically needed in the first 3 years, every 6 months in the following 2 years, and annually thereafter. Each follow-up examination will include a complete history and physical examination, contrast-enhanced MRI or CT (only if MRI is contraindicated), and blood tests including complete blood counts, serum electrolytes, liver/renal function tests, and EBV DNA copies. PET/CT scans, bone scans, thoracic $\mathrm{CT}$, and abdominal ultrasound or $\mathrm{CT}$ 
are optional based on the clinical findings at follow-up examinations.

\subsubsection{Treatment at tumor progression}

Further treatments after the completion of CIRT may be clinically needed in cases of tumor recurrence or progression. Surgical resection or a third course of radiation for locoregional recurrence, systemic treatment with chemotherapy or targeted therapy may be considered for distant metastasis.

\subsection{Statistics}

\section{Phase I}

The primary objective is to determine the CIRT dose that is associated with a dose-limiting adverse toxicity (DLT) in $<25 \%$ of patients with locally recurrent NPC previously treated with high-dose photon radiation. DLT is defined as any Grade 4 toxicity according to CTCAE Version 4.03, possibly, probably or definitely associated to CIRT that occurred within 6 months after the completion of re-irradiation using CIRT. Treatment induced toxicity also includes deterioration in performance status to $\geq 3$ developing during or within the 6 months following completion of CIRT. The secondary objective of the phase I part of the trial is overall survival (OS) and local progress-free survival (LPFS). The maximal tolerance dose (MTD) of CIRT for re-irradiation will be determined at the end of Phase I.

Dose levels of CIRT were assigned based on the Time-to-Event Continual Reassessment Method (TITE-CRM) algorithm ${ }^{21}$. This probability of DLT is continually updated during the trial using data from all enrolled patients and their updated outcome. Patients with partial follow-up at a time of a new enrollment (i.e., $<6$ months) are weighted by the proportion of the follow-up time completed. Newly enrolled patients are assigned to the dose estimated to have a probability closest but not higher than the DLT probability of 0.25 . Four patients will be entered to the starting dose level. Prior to escalation, at least one patient must have completed the full observation period (6 months) at the previous level without DLT. Dose escalation was restricted to one level between any 2 patients. The dose increases until DLT is observed or when 65GyE is reached.

Six dose levels and their initial estimation of the probability for DLT (pDLT) are planned within the Phase I part (Table 1). We estimate that 2 patients will be accrued each month.

The phase I part of the trial was designed to accrue up to 25 patients. Patients completed $\geq 90 \%$ of the planned CIRT dose are considered evaluable. Patients who completed $\geq 90 \%$ planned CIRT but became un-evaluable for 6 months are counted as evaluable in the final analysis, and weighted by the proportion of the observation period for which they were evaluable. Replacements of any accrued patients can only be considered for patients who did not complete therapy for reasons other than toxicity.

A simple two-parameter logistic regression model is used to estimate the pDLT at each dose level at the end of the trial. Secondary endpoints are response of tumor to CIRT, local progression free survival, overall survival, as well as other toxicity and safety data on the studied dose levels. The Kaplan-Meier method will be used to summarize OS and LPFS for all patients in the trial. The software package SAS (V9.1, Cary, NC, USA) is used for statistical analyses.

\section{Phase II}

The primary objective of the Phase II part of the current trial is to evaluate the 24 month overall survival (OS) rate $\Pi$ for patients with locally recurrent NPC receiving CIRT. According to results reported in the literature, the 24 month OS rate for patients with locally advanced recurrent NPC (rT3 or rT4 lesions) treated with IMXT or proton therapy range between $40 \sim 50 \%$ 5,6,7. We expect that CIRT can improve the OS at 2 years by $20 \%$. Thus, the confirmatory analysis of the primary endpoint assesses the following test problem: $\mathrm{H} 0: \Pi \leq 0.50=\Pi 0$ versus $\mathrm{H} 1: \Pi>0.70$.

As this trial is the first study to address the efficacy of re-irradiation with CIRT on locally recurrent NPC, the expected OS rate of $70 \%$ at 2 years represent an estimate based on the best of the researchers' knowledge. We use the exact single-stage design for the Phase II part of the study ${ }^{22}$. The null hypothesis that the true OS rate is $50 \%$ will be tested against a one-sided alternative. A total of 37 patients will be needed and the null hypothesis will be rejected if 23 or more survivors are observed in 37 patients with a median follow up of 2 years. This design yields a type I error rate of 0.05 and power of 0.8 when the true OS rate is $70 \%$.

For ethical reasons, all patients treated with the recommended dose of CIRT in the Phase I part of the study will be included in the Phase II part to minimize the number of patients accrued to the trial. As such, the required sample size of the second phase and the entire study can be reduced accordingly.

\section{Data Collection/Safety/Management and Ethical/Legal Aspects}

\subsection{Data safety monitoring board}

The Institution Review Board (IRB) of SPHIC will act as the independent data safety monitoring board to monitor the recruitment, the report of 
adverse events, and the data quality semi-annually. Based on the results of the review, the IRB will provide the Principal Investigator (PI) with requirements and recommendations on modification of the trial, which may or may not include termination of the trial.

\subsection{Data collection and management}

Based on the requirement of the Chinese GCP-Regulation, documents of the trial will be maintained for at least 5 years after the completion of the trial. The Research Unit of the Medical Affair Department of the SPHIC will be responsible for archiving all relevant data of the trial. Medical charts of the patients including all imaging studies are required to be archived for at least 7 years based on the local legal requirements.

\subsection{Ethical and legal aspects}

Approval by the IRB of SPHIC was obtained on September 17, 2015. The accrual of patients will be started after September 18, 2015.

The protocol will be conducted according the guidelines of Good Clinical Practice (GCP) of China and the principles of the Declaration of Helsinki (2008 Version adopted at the 59th WMA General Assembly, Seoul, October 2008).

\section{Discussion}

Treatment of locally recurrent NPC poses a major challenge for oncologists specialized in head and neck malignancies. A number of radiation techniques have been used and studied, but outcomes from re-irradiation using IMXT, SRS/SBRT, or brachytherapy have been suboptimal. Limited ranges of effective dose coverage is the main pitfall of brachytherapy and SRS for large lesions in the base of skull. Although IMXT and SBRT can be used to treat more bulky lesions, re-irradiation of the surrounding normal tissues that are previously damaged by high-dose RT may cause severe or fatal late toxicities such as mucosal ulceration or bleeding 5,6,7. In addition, recurrent malignancies after previous high-dose IMXT may harbor cells that are highly resistant to photon radiotherapy delivered in conventional fractionation ${ }^{23}$. Clearly, novel and more effective treatment approaches are needed.

CIRT is a highly precise radiation therapy technique due to its physical properties. It also provides higher relative biological effectiveness (RBE) that can be used for the treatment of malignancies that are resistant to photon radiation such as those which recurr after previous high-dose IMXT. Improved outcomes using CIRT as compared to re-irradiation using photon or proton have been demonstrated for a number of malignancies ${ }^{24}$. Although the RBE for NPC cells have not been determined, preclinical data have demonstrated that the RBE ranges from 2-5 for radiation-resistant cells such as cells hepatocellular carcinoma, melanoma or GBM cell lines $25,26,27$. In addition, early results from clinical studies have suggested that such advantage might translate into improved clinical outcomes ${ }^{28}$.

Early clinical data on CIRT in re-irradiation of locally recurrent malignancies including those recurred in the base of skull after previous high-dose radiation have demonstrated overall safety and efficacy ${ }^{17}$. However, no patients with recurrent NPC had been included in those studies. Early clinical experience at the SPHIC indicated that the patients treated with intensity-modulated carbon ion radiotherapy (IMCT) for locally recurrent NPC to 50 55GyE in 20 25 fractions produced a response rate (defined PR+CR) of $100 \%$ without radiation-induced adverse effects of Grade 2 or above at 6-8 weeks of follow-up. Nevertheless, the optimal dose, efficacy, and safety of carbon ion RT delivered to treatment-resistant tumor, which are surrounded with pharyngeal mucosa that has been previously irradiated to definitive doses need to be addressed in a prospective fashion.

As such, the present trial evaluates IMCT using pencil-beam scanning technique delivered as re-irradiation in patients with locally recurrent NPC. In the first phase of the study, a dose escalation will be performed to explore the optimal dose of IMCT that can be prescribed for recurrent NPC lesions in the post-nasal space. Thereafter, the second phase of the trial will study the efficacy of IMCT at MTD as definitive treatment for patients with locally recurrent NPC.

\section{Acknowledgements}

Funding sources: Shanghai Hospital Development Center (Joint Breakthrough Project for New Frontier Technologies. Project No. SHDC 12015118); Science and Technology Commission of Shanghai Municipality (Project No. 15411950102 \& 15411950106); Natural Science Foundation of Shanghai (Project No. 14ZR1407100).

\section{Statement of ethical standards}

IRB approval was obtained for this trial, and the trial will be performed in accordance with the ethical standards laid down in the 1964 Declaration of Helsinki and its later amendments. All persons will give their informed consent prior to their inclusion in the study. 


\section{Conflict of interest}

All authors declare that they have no conflict of interest.

\section{References}

1. Wang TJC, Riaz N, Cheng SK, et al. Intensity-modulated radiation therapy for nasopharyngeal carcinoma: a review. J Radiat Oncol. 2012; 1(2): 129-46.

2. Wei WI, Chan JY, Ng RW, Ho WK. Surgical salvage of persistent or recurrent nasopharyngeal carcinoma with maxillary swing approach - Critical appraisal after 2 decades. Head Neck. 2011 Jul; 33(7): 969-75.

3. Chua DT, Wei WI, Sham JS, et al. Stereotactic radiosurgery versus gold grain implantation in salvaging local failures of nasopharyngeal carcinoma. Int J Radiat Oncol Biol Phys. 2007 Oct 1; 69(2):469-74.

4. Leung TW, Tung SY, Sze WK, et al. Salvage brachytherapy for patients with locally persistent nasopharyngeal carcinoma. Int J Radiat Oncol Biol Phys. 2000 May 1; 47(2): 405-12

5. Hua YJ, Han F, Lu LX, et al. Long-term treatment outcome of recurrent nasopharyngeal carcinoma treated with salvage intensity modulated radiotherapy. Eur J Cancer. 2012 Dec; 48(18):3422-8.

6. Han F, Zhao C, Huang SM, et al. Long-term outcomes and prognostic factors of re-irradiation for locally recurrent nasopharyngeal carcinoma using intensity-modulated radiotherapy. Clin Oncol (R Coll Radiol). 2012 Oct; 24(8):569-76.

7. Qiu S, Lin S, Tham IW, et al. Intensity-modulated radiation therapy in the salvage of locally recurrent nasopharyngeal carcinoma. Int J Radiat Oncol Biol Phys. 2012 Jun 1;83(2):676-83.

8. Pryzant RM, Wendt CD, Delclos L, Peters LJ. Re-treatment of nasopharyngeal carcinoma in 53 patients. Int J Radiat Oncol Biol Phys. 1992; 22(5):941-7.

9. Brown AP, Urie MM, Chisin R, Suit HD. Proton therapy for carcinoma of the nasopharynx: a study in comparative treatment planning. Int J Radiat Oncol Biol Phys. 1989 Jun; 16(6): 1607-14.

10. Feehan PE, Castro JR, Phillips TL, et al. Recurrent locally advanced nasopharyngeal carcinoma treated with heavy charged particle irradiation. Int J Radiat Oncol Biol Phys. 1992; 23(4):881-4.

11. Lin R, Slater JD, Yonemoto LT, et al. Nasopharyngeal carcinoma: repeat treatment with conformal proton therapy--dose-volume histogram analysis. Radiology. 1999 Nov; 213(2):489-94

12. Huang YW, Pan CY, Hsiao YY, et al. Monte Carlo simulations of the relative biological effectiveness for DNA double strand breaks from $300 \mathrm{MeV} \mathrm{u(-1)}$ carbon-ion beams. Phys Med Biol. 2015 Aug 7;60(15):5995-6012.

13. Schulz-Ertner D, Nikoghosyan A, Didinger B, et al. Therapy strategies for locally advanced adenoid cystic carcinomas using modern radiation therapy techniques. Cancer. 2005; 104: 338-44

14. Schulz-Ertner D, Nikoghosyan A, et al. Carbon ion radiotherapy of skull base chondrosarcomas. Int J RadiatOncolBiol. 2007; 67:171-7

15. Schulz-Ertner D, Karger CP, Feuerhake A, et al. Effectiveness of carbon ion radiotherapy in the treatment of skullbase chordomas. Int J RadiatOncolBiol. 2007; 68:449-57.

16. Combs SE, Nikoghosyan A, Jaekel O, et al. Carbon ion radiotherapy for pediatric patients and young adults treated for tumors of the skull base. Cancer. 2009; 115:1348-55.

17. Combs SE, Kalbe A, Nikoghosyan A, et al. Carbon ion radiotherapy performed as re-irradiation using active beam delivery in patients with tumors of the brain, skull base and sacral region. Radiother Oncol. 2011 Jan; 98(1):63-7

18. Cheung YK, Chappell R. Sequential designs for phase I clinical trials with late-onset toxicities. Biometrics. 2000 Dec; 56(4):1177-82.

19. Normolle D, Lawrence T. Designing dose-escalation trials with late-onset toxicities using the time-to-event continual reassessment method. J Clin Oncol. 2006 Sep 20; 24(27):4426-33.

20. Emami B, Lyman J, Brown A, et al. Tolerance of normal tissue to therapeutic irradiation. Int J Radiat Oncol Biol Phys. 1991; 21:109-22.

21. Cheung YK, Chappell R. Sequential designs for phase I clinical trials with late-onset toxicities. Biometrics. 2000 Dec; 56(4):1177-82.

22. A'Hern RP. Sample size tables for exact single-stage phase II designs. Stat Med. 2001 Mar 30;20(6):859-66.

23. Kong L, Lu JJ. Reirradiation of locally recurrent nasopharyngeal cancer: history, advances, and promises for the future. Chin Clin Oncol. 2016; Accepted

24. Schulz-Ertner D, Tsujii H: Particle radiation therapy using proton and heavier ion beams. J Clin Oncol. 2007; 25:953-64.

25. Iwadate Y, Mizoe J, Osaka Y, Yamaura A, Tsujii H. High linear energy transfer carbon radiation effectively kills cultured glioma cells with either mutant or wild-type p53. Int J Radiat Oncol Biol Phys. 2001; 50:803-8.

26. Combs SE, Bohl J, Elsasser T, et al. Radiobiological evaluation and correlation with the local effect model (LEM) of carbon ion radiation therapy and temozolomide in glioblastoma cell lines. Int J Radiat Biol. 2009 Feb; 85(2):126-37.

27. Calipel A, Lux AL, Guérin S, et al. Differential radiosensitivity of uveal melanoma cell lines after X-rays or carbon ions radiation. Invest Ophthalmol Vis Sci. 2015 May 1;56(5):3085-94.
28. Habermehl D, Wagner M, Ellerbrock $M$, et al. Reirradiation using carbon ions in patients with locally recurrent rectal cancer at HIT: First results. Ann Surg Oncol. 2015 Jun;22(6):2068-74 